\title{
Interactive Volumetric Lighting Simulating Scattering and Shadowing
}

\author{
Timo Ropinski* \\ University of Münster
}

\author{
Christian Döring ${ }^{\dagger}$ \\ European Institue of Molecular Imaging (EIMI)
}

\author{
Christof Rezk-Salama \\ Mediadesign University of \\ Applied Sciences, \\ Düsseldorf
}
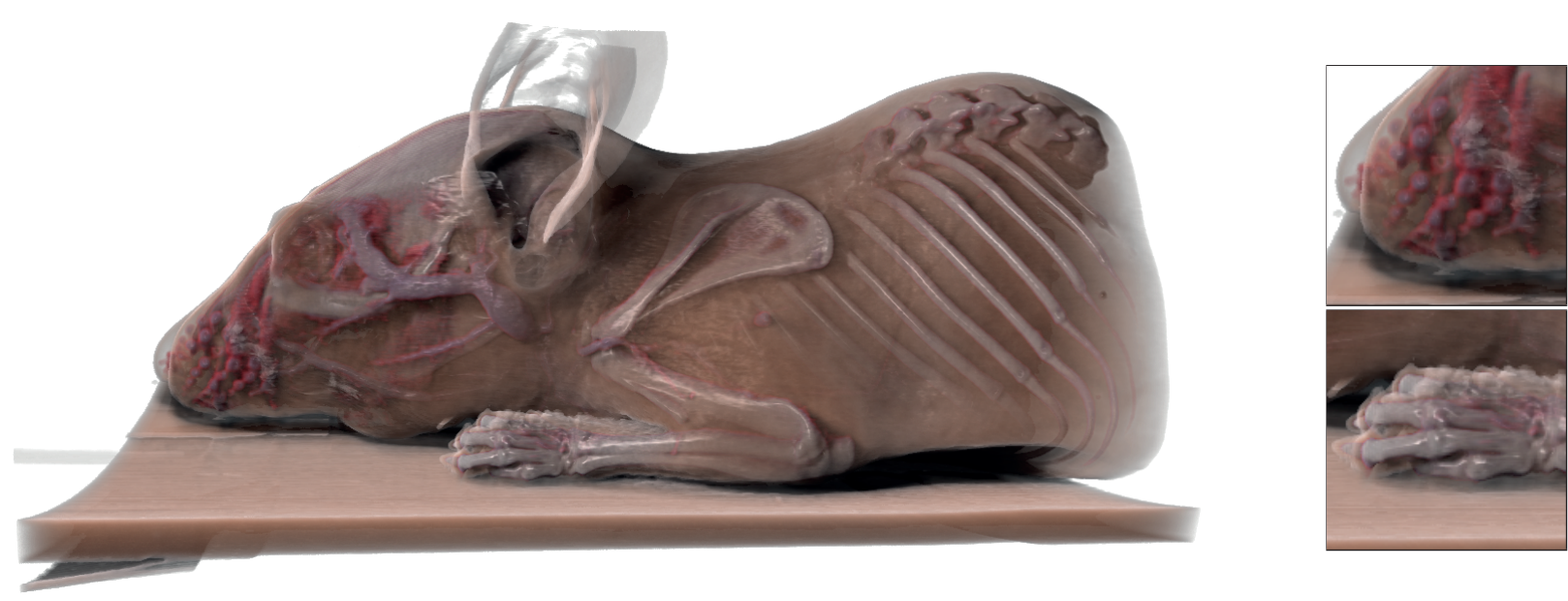

Figure 1: A CT scan of a mouse $(360 \times 270 \times 550$ voxels $)$ rendered with the proposed illumination model. The closeups on the right show, that semi-transparent, diffuse as well as specular materials are captured realistically.

\begin{abstract}
In this paper we present a volumetric lighting model, which simulates scattering as well as shadowing in order to generate high quality volume renderings. By approximating light transport in inhomogeneous participating media, we are able to come up with an efficient GPU implementation, in order to achieve the desired effects at interactive frame rates. Moreover, in many cases the frame rates are even higher as those achieved with conventional gradientbased shading. To evaluate the impact of the proposed illumination model on the spatial comprehension of volumetric objects, we have conducted a user study, in which the participants had to perform depth perception tasks. The results of this study show, that depth perception is significantly improved when comparing our illumination model to conventional gradient-based volume shading. Additionally, since our volumetric illumination model is not based on gradient calculation, it is also less sensitive to noise and therefore also applicable to imaging modalities incorporating a higher degree of noise, as for instance magnet resonance tomography or $3 \mathrm{D}$ ultrasound.
\end{abstract}

Index Terms: I.3.7 [Computer Graphics]: Three-Dimensional Graphics and Realism-Color, shading, shadowing, and texture.

\section{INTRODUCTION}

Today, volume rendering can be performed in real-time on consumer graphics hardware, thanks to a variety of acceleration techniques developed in the past. In practice, the shading in most

*e-mail: ropinski@math.uni-muenster.de

†e-mail:christian.doering@uni-muenster.de

‡e-mail:c.rezk-salama@mediadesign-fh.de cases is rather simple, i. e., gradient-based local Phong illumination, which comes with two major drawbacks. First, since the Phong illumination model has been originally developed for surface lighting, it relies on a well-defined gradient as substitute for the surface normal when applied to volume data. Second, except for the voxels used for the gradient estimation, neighborhood information is not taken into account. This restricts the model to the simulation of local illumination phenomena, although advanced illumination models would significantly improve spatial comprehension [12].

While normalized gradients are usually a good approximation for the normal vectors of isosurfaces in CT data, modalities with a significantly lower signal-to-noise ratio will lead to severe degradation of the gradient quality. This is not only the case for more recently emerging modalities as 3D ultrasound (US) or positron emission tomography, but also for very well established modalities such as magnet resonance tomography (MRT). While gradient filtering is frequently applied when shading such modalities, its application is limited due to the a loss of information and the performance penalty induced by on the fly gradient estimation. It is thus desirable to develop a volumetric illumination model providing good perceptual qualities without relying on gradient computation.

A gradient vector can be thought of as a very rough approximation of the vicinity of a voxel, where only 6 to 26 direct neighbors are taken into account. Due to this small number of contributing voxels, noise has a rather strong impact. Incorporating a larger neighborhood around the current voxel would improve the estimate. In this sense, the extreme case would be to consider the entire data set, which would pave the way for the integration of global illumination effects. The approach of incorporating a larger neighborhood into the shading computation is not new and has been previously exploited by other shading models $[11,20,23]$. In this paper, we follow this idea and present a physically plausible volumetric illumination model with a flexibility which meets the perceptual requirements of the user. Figure 1 shows the application of the proposed volumetric illumination model to a CT scan of a mouse. As 
it can be seen, semi-transparent as well as diffuse and specular materials are rendered realistically.

The contributions of this paper are as follows. In order to generate a realistic representation of participating media for the human observer, the integration of spatially varying scattering effects is supported. Since interactive high-quality volume rendering is desired, we aim at an integration of these effects into GPU-based volume ray-casting. A perceptional problem of scattering effects for visualization is the vanishing of hard shadow borders, which are important for the spatial comprehension of a scene [26]. In our model the shadow computation is decoupled from the scattering, which allows substantially harder shadow borders. Many previous solutions to volumetric scattering lead to unrealistic appearance of surfaces within the volume data. Bone structures in CT often look like marble when scattering is applied. Our model allows us to easily control the amount of surface-like reflection and volumetric scattering. While the approximative nature of our model leads to physically plausible results, the solution to the light transport equation cannot be considered as physically correct. Therefore, we have conducted a user study to assess the perceptual qualities of our volumetric illumination model. The obtained results indicate, that our model significantly improves depth perception, i. e., leading to faster and less error prone depth decisions when compared to gradient-based Phong illumination. On top of that, our performance measurements show that in many cases even an increase in performance can be achieved compared to gradient-based lighting.

To the best of our knowledge, the developed illumination model is the first one, which meets all the described requirements at the same time while preserving full interactivity and providing good perceptual qualities.

In the remainder of this paper, we first discuss related work in Section 2, before deriving the interactive volumetric illumination model supporting scattering as well as shadowing in Section 3. The technical realization allowing interactive frame rates is described in the following two sections, whereby Section 4 explains the lighting computation and Section 5 includes details about the actual rendering. Section 6 demonstrates some applications of the developed illumination model and discusses the results of the conducted user study, before the paper concludes in Section 7.

\section{Related Work}

In his seminal paper on optical models for volume rendering, Max [16] emphasizes the importance of global illumination effects. Accurate solutions of the physical equations of light scattering in participating media, however, are still too expensive for real-time applications.

Originally developed for surface lighting, ambient occlusion [29] and obscurance shading [17] have become popular in volume rendering. These techniques have been applied successfully to enhance the perception of spatial relationships for isosurface rendering $[1,24,18]$. Several techniques exist to calculate ambient occlusion for direct volume rendering. Hernell et al. [7, 8] and Ljung et al. [14] propose local ambient occlusion as a fast way to calculate soft shadows for volumetric data with varying transfer functions. Ropinski et al. [20] calculate dynamic updates for both ambient occlusion and scattering effects by utilizing local histograms. Schott et al. [23] propose a different, but also inexpensive implementation which restricts ambient occlusion computation to a user-specified cone angle by assuming the light position coincides with the camera position (head light). The vicinity occlusion map proposed by Diaz et al. [9] is an adoption of screen-space ambient occlusion for direct volume rendering. A significant drawback of this postprocessing technique is its dependency on a valid depth buffer, which restrict itself to rather opaque renditions. The obscurance shading technique proposed by Ruiz et al. [21] is a generalization of ambient occlusion for illustrative purposes instead of soft shadows.
Since ambient occlusion techniques simulate the appearance of soft shadows assuming ambient light only, they cannot account for external light sources.

To incorporate external light sources, different approaches have been proposed. A texture-slicing techniques which allows for shadows caused by attenuation of one distant light source has been proposed by Behrens and Ratering [2], who superimpose a separate shadow volume on top of the scalar data set. While our approach is similar in spirit, it also supports scattering and can be applied to point light sources without posing constraints on the light position. This allows an interactive changing of the light position, for instance in order to achieve a top-left lighting with supports resolving convex-concave ambiguities and is therefore a preference in illustrations [3, 25]. Zhang et al. [27, 28] have proposed methods to incorporate shadows into sheet-based splatting techniques, which are less efficient compared to texture slicing or GPU-based volume ray-casting. Desgranges et al. [4] propose a gradient-free technique for shading and shadowing which produces visually pleasing shadowing effects. In the strict sense, however, it is accurate only for a head light, but in this case no shadows would be visible at all. For GPU-based volume raycasting, Ropinski et al. [19] give an overview of different possibilities to incorporate shadows from external light sources. The most prominent implementation is shown by Hadwiger et al. [6] who demonstrate a GPU-based implementation of deep shadow maps with real-time capabilities.

In comparison to shadowing algorithms, much less work has been done in order to support scattering. Dobashi et al. [5] have proposed a hardware-accelerated rendering technique to account for atmospheric scattering effects in homogeneous participating media. To account for scattering of light in volume data, Kniss et al. $[10,11]$ have proposed a half-angle slicing technique which allows textured slices to be rendered from both light and viewing direction simultaneously. They account for scattering effects in slicebased volume rendering by sampling the incident light from multiple directions while updating the light's attenuation map. Similar to our implementation, scattering is restricted to a user-specified cone which represents a special type of phase function. We emphasize the importance of this publiaction, however, while the results show convincing light transport effects, their implementation is inconsistent in the strict sense: It accounts for light scattered around the dominant direction of incident light, but neglects scattering of outgoing light on its way towards the eye, which can be incorporated in our model. Furthermore, the technique can be only applied to slice-based volume rendering. Rezk-Salama [22] has proposed a fast Monte-Carlo-based algorithm for volumetric rendering, however, with scattering events restricted to a limited set of isosurfaces.

To these ends we propose a flexible optical model which incorporates scattering, shadowing and specular reflections, and we demonstrate its efficient implementation based on GPU-based volume ray-casting. Our technique is flexible in the sense that it may be used to model scattering more physically accurate compared to previous work, but also allows us to ease the restrictions of physical laws by decoupling shadow calculation from scattering.

\section{Volumetric Illumination Model}

Scattering and shadowing have a great influence on the quality of volume rendered images. The radiance $L_{S}\left(\vec{x}, \vec{\omega}_{o}\right)$ which is scattered from position $\vec{x}$ inside the volume in direction $\vec{\omega}_{o}$ can be defined as:

$$
L_{s}\left(\vec{x}, \vec{\omega}_{o}\right)=s\left(\vec{x}, \vec{\omega}_{i}, \vec{\omega}_{o}\right) \cdot L_{i}\left(\vec{x}, \vec{\omega}_{i}\right)+L_{e}(\vec{x}),
$$

where $L_{i}\left(\vec{x}, \vec{\omega}_{i}\right)$ is the incident radiance reaching point $\vec{x}$ from direction $\vec{\omega}_{i}, L_{e}(\vec{x})$ is the emissive radiance and $s\left(\vec{x}, \vec{\omega}_{i}, \vec{\omega}_{o}\right)$ describes the actual shading, which is dependent on both the incident light direction $\vec{\omega}_{i}$ as well as the outgoing light direction $\vec{\omega}_{o}$. Furthermore, $s\left(\vec{x}, \vec{\omega}_{i}, \vec{\omega}_{o}\right)$ is dependent on parameters, which may vary based on $\vec{x}$, as for instance the optical properties assigned through the transfer 
function. In the context of volume rendering, $s\left(\vec{x}, \vec{\omega}_{i}, \vec{\omega}_{o}\right)$ is often written as:

$$
s\left(\vec{x}, \vec{\omega}_{i}, \vec{\omega}_{o}\right)=\tau(\vec{x}) \cdot p\left(\vec{x}, \vec{\omega}_{i}, \vec{\omega}_{o}\right),
$$

where $\tau(\vec{x})$ represents the extinction coefficient at position $\vec{x}$ and $p\left(\vec{x}, \vec{\omega}_{i}, \vec{\omega}_{o}\right)$ is the phase function describing the scattering characteristics of the participating medium at the position $\vec{x}$. When considering shadowing in this model, where the attenuation of external light traveling through the volume is incorporated, the incident radiance $L_{i}\left(\vec{x}, \vec{\omega}_{i}\right)$ can be defined as:

$$
L_{i}\left(\vec{x}, \vec{\omega}_{i}\right)=L_{l} \cdot T\left(\vec{x}_{l}, \vec{x}\right) .
$$

This definition is based on the standard volume rendering integral, where the light source is located at $\vec{x}_{l}$ and has the radiance $L_{l}$ and $T\left(\vec{x}_{l}, \vec{x}\right)$ is the corresponding transparency between $\vec{x}_{l}$ and $\vec{x}$.

According to Max, the standard volume rendering integral simulating absorption and emission can be extended with these definitions to support single scattering and shadowing:

$$
\begin{aligned}
& L\left(\vec{x}, \vec{\omega}_{o}\right)=L_{0} \cdot T\left(\overrightarrow{x_{0}}, \vec{x}\right) \\
& \quad+\int_{\overrightarrow{x_{0}}}^{\vec{x}}\left(L_{e}(\vec{x})+\left(s\left(\vec{x}^{\prime}, \vec{\omega}_{i}, \vec{\omega}_{o}\right) \cdot L_{i}\left(\vec{x}^{\prime}, \vec{\omega}_{i}\right)\right)\right) \cdot T\left(\vec{x}^{\prime}, \vec{x}\right) d \vec{x}^{\prime}
\end{aligned}
$$

with $\vec{\omega}_{i}=\vec{x}_{l}-\vec{x}^{\prime}, L_{0}$ being the background intensity and $\vec{x}_{0}$ a point behind the volume. When extending this equation to support multiple scattering, it is necessary to integrate the scattering of light coming from all possible directions $\vec{\omega}_{i}$ on the unit sphere. However, Max has stated that this would be overkill for most scientific visualization applications [16]. Similar to Kniss et al. [11] we incorporate indirect lighting by blurring the incoming light within a given cone centered about the incoming light direction. In contrast to their technique, our approach can be easily extended, to also account for scattering that happens when the light travels towards the eye. To achieve this, we use an additional scattering pass on the light volume, which blurs the outgoing light within a cone facing away from the viewing direction. Another important difference compared to [11] is that we use separate blurring for the chromaticity and the intensity of the light (luminance). Although such an approach is not physically correct, the decoupling of shadow computation from color bleeding provides us with additional flexibility to optimize the perception of spatial structures, which is known to be improved, when hard shadow borders are present [26], since humans are very sensitive in perceiving small changes in luminance, but not in hue [13]. To guarantee hard shadow borders, we omit the blurring of the intensity part and just apply standard linear interpolation. Thus, our illumination model can be specified as follows:

$$
L\left(\vec{x}, \vec{\omega}_{o}\right)=L_{0} \cdot T\left(\overrightarrow{x_{0}}, \vec{x}\right)+\int_{\overrightarrow{x_{0}}}^{\vec{x}}\left(L_{e}(\vec{x})+q\left(\vec{x}, \vec{\omega}_{i}, \vec{\omega}_{o}\right)\right) \cdot T\left(\vec{x}^{\prime}, \vec{x}\right) d \vec{x}^{\prime},
$$

where the radiance $q\left(\vec{x}, \vec{\omega}_{i}, \vec{\omega}_{o}\right)$ is calculated as the transport color $t(\vec{x})$, as assigned through the transfer function, multiplied by the chromaticity $c\left(\vec{x}, \vec{\omega}_{o}\right)$ of the in-scattered light and modulated by the attenuated luminance $l_{i}\left(\vec{x}, \vec{\omega}_{i}\right)$ :

$$
q\left(\vec{x}, \vec{\omega}_{i}, \vec{\omega}_{o}\right)=t(\vec{x}) \cdot c\left(\vec{x}, \vec{\omega}_{o}\right) \cdot l_{i}\left(\vec{x}, \vec{\omega}_{i}\right) .
$$

The transport color $t(\vec{x})$ as well as the chromaticity $c\left(\vec{x}, \vec{\omega}_{o}\right)$ are of course wave length dependent, while the scalar $l_{i}\left(\vec{x}, \vec{\omega}_{i}\right)$ describes the incident (achromatic) luminance. Multiplying all together will result in the incident radiance. Chromaticity $c\left(\vec{x}, \vec{\omega}_{0}\right)$ is computed from the in-scattered light

$$
c\left(\vec{x}, \vec{\omega}_{o}\right)=\int_{\Omega} \tau(\vec{x}) \cdot p\left(\vec{x}, \vec{\omega}_{i}^{\prime}, \vec{\omega}_{o}\right) \cdot c\left(\vec{x}, \vec{\omega}_{i}^{\prime}\right) d \vec{\omega}_{i}^{\prime},
$$

where $\Omega$ is the unit sphere centered around $\vec{x}$. In our model, $p\left(\vec{x}, \vec{\omega}_{i}{ }^{\prime}, \vec{\omega}_{o}\right)$ is a strongly forward peaked phase function:

$$
p\left(\vec{x}, \vec{\omega}_{i}^{\prime}, \vec{\omega}_{o}\right)=\left\{\begin{array}{cl}
C \cdot\left(\vec{\omega}_{i}{ }^{\prime} \cdot \vec{\omega}_{o}\right)^{\beta} & \text { if } \vec{\omega}_{i}{ }^{\prime} \cdot \vec{\omega}_{o}<\theta(\vec{x}) \\
0 & \text { otherwise. }
\end{array}\right.
$$

Similar to the work of Kniss et al. [11], the cone angle $\theta$ is used to control the amount of scattering and depends on the scalar data set at position $\vec{x}$. It is higher for less opaque voxels and can be maximal $\frac{\pi}{4}$. The phase function is a Phong lobe whose extent is controlled by the exponent $\beta$, restricted to the cone angle $\theta$. The constant $C$ must be chosen with respect to $\beta$ to ensure energy conservation. If the gradient magnitude at $\vec{x}$ is high with respect to a user-specified threshold, we have a well-defined gradient and may as well compute specular reflections. This allows us to account for both surface like reflection and volumetric scattering.

\section{Light Volume Calculation}

To efficiently approximate the volumetric illumination model described in the previous section, we propose an algorithm, which exploits the features of current GPUs. While in standard DVR, the final color is obtained by incorporating emission and absorption along a viewing ray, we exploit an alternative concept in order to independently compute the scattering and the shadowing contribution. When considering just shadowing, a straight forward extension to a GPU-based volume ray-caster already requires a substantial overhead during rendering. It would require at least 1 shadow ray per sample. Since the number of samples directly influences the image quality it is often rather high. When for instance considering a given image resolution of $1024^{2}$ and a volumetric data set having a resolution of $512^{3}$ voxels, due to the Nyquist theorem $O\left((512 \cdot 2) \cdot 1024^{2}=1024^{3}=n_{s r}\right)$ shadow rays would be required. When taking into account, that each of these $n_{s r}$ shadow rays needs to be sampled sufficiently high, it becomes clear that this shadowing approach results in a high performance penalty. When additionally simulating scattering by computing different ways of light through the medium, it would become even more expensive. Therefore, we have decided to realize the derived volumetric illumination model not in image space, where a high number of sampling operations is necessary, but directly in volume space.

To compute the chromaticity $c\left(\vec{x}, \vec{\omega}_{o}\right)$, we process the volume in front-to-back order from the light position and blur the chromaticity of the incident light within a cone centered around the light direction, with the tip of the cone pointing away from the light source, while we directly accumulate the opacity in order to compute the luminance $l_{i}\left(\vec{x}, \vec{\omega}_{i}\right)$. To also simulate the scattering, that occurs when light travels towards the eye, we can process the light volume again in back-to-front order with respect to the viewing position and blur the outgoing light within a cone centered around the viewing direction, with the tip pointing towards the eye position. By integrating this additional pass, pitch black shadows can be avoided in some areas resulting in a more vivid image.

Thus, the additional performance impact becomes independent of the sampling rate, and shadowing as well as scattering can be realized with only $O(v \cdot a)$ sampling operations, where $v$ is the number of voxels and $a$ the size of the desired neighborhood used for blurring the indirect achromatic light contribution. In the following subsections, we will explain our voxel space realization of the described volumetric illumination model.

\subsection{Light Propagation}

The proposed realization is based on an illumination volume. Alternatively, one could think about using a deep shadow map approach [15]. However, while deep shadow maps would allow a similar shadow impression and do not need to store a volumetric data set, they do not allow an easy integration of scattering and 


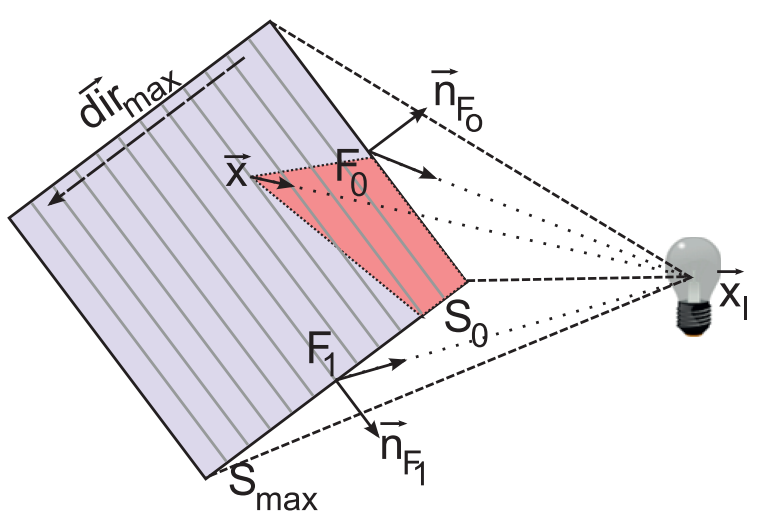

Figure 2: Light is propagated through the volume slice by slice starting at the cube face $F_{0}$ being closest to the light source located in $\overrightarrow{x_{l}}$.

have the drawback, that they incorporate a data set dependent user defined error value. In contrast our technique does not need any additional user assigned parameters.

Our voxel space light propagation model is similar in spirit to the shadowing approach presented by Behrens and Ratering [2]. However, in contrast their approach is constrained to simulate shadows with one distant light source hitting the volume at an angle of $45^{\circ}$. Our technique is not confined in this way and therefore allows us to capture shadowing of arbitrary directional or external point light sources as well as scattering effects, leading to more realistic rendering results. In this subsection, we will explain our technique for an arbitrary point light source.

In order to exploit the features of current GPUs, we approximate the described model by processing the volumetric data set slice by slice. Thus, we compute an illumination volume, which is updated on the fly whenever the transfer function and/or the light position $\vec{x}_{l}$ has been changed. Since the shadowing as well as the scattering effects at each position $\vec{x}$ depend only on those voxels lying in between $\vec{x}$ an $\vec{x}_{l}$, it is sufficient to process the volumetric data set starting near $\vec{x}_{l}$ and proceeding in a direction facing away from the light source to compute $c\left(\vec{x}, \vec{\omega}_{o}\right)$ and $l_{i}\left(\vec{x}, \vec{\omega}_{i}\right)$. We start at the cube face $F_{0}$ being closest to the light source and propagate illumination information along one of the major axes of the volume, i. e., $x, y$ or $z$. As illustrated in Figure 2, the used axis is determined by the principle processing direction $d_{i{ }_{\text {max }}}$. Determining $d i \vec{r}_{\text {max }}$ can be thought of choosing the $F_{j}$ of all cube faces $F$, which has the largest projection as seen from $\vec{x}_{l}$. Since, we want to propagate the lighting information from $\vec{x}_{l}$ through the volume, not all $F_{j}$ come into account, but only those which are visible from $\vec{x}_{l}$ and thus fulfill the equation $\overrightarrow{n_{F_{j}}} \cdot\left(\overrightarrow{x_{l}}-\overrightarrow{c_{F_{j}}}\right)<0$, where $\overrightarrow{n_{F_{j}}}$ is the normal and $\overrightarrow{c_{F_{j}}}$ is the center of $F_{j}$. Based on this observation, we sort the three cube faces being visible from $\vec{x}_{l}$, such that $F_{0}$ minimizes $\overrightarrow{n_{F}} \cdot\left(\overrightarrow{x_{l}}-\overrightarrow{c_{F_{j}}}\right)$ (see Figure 2). Thus $F_{0}$ is the cube face with the largest and $F_{2}$ is the cube face with the smallest projection as seen from $\vec{x}_{l}$. Since $\vec{n}_{F_{0}}$ is the normal of $F_{0}$, the principle light propagation direction $\operatorname{dir}_{\max }$ can be set to $d i \vec{r}_{\text {max }}=-\overrightarrow{n_{F_{0}}}$.

Now, that we know in which direction we have to propagate the light information through the volume, we can start at the slice $S_{0}$ which is closest to $\vec{x}_{l}$ along the axis $d i \vec{r}_{\text {max }}$. During the light propagation we step through all slices $S_{i}$ until we get to the last slice $S_{\max }$. This propagation is performed on the fly by exploiting framebuffer objects and render-to-texture functionality. Since rendering into a 3D texture only allows to render along the $z$-direction, we have to apply an axis permutation during the light propagation, in order to support arbitrary light positions $\vec{x}_{l}$. However, for explanatory rea- sons, we first describe the simple case, where $d i \vec{r}_{\max }$ is along the positive or negative $z$-axis, before briefly describing the permutation, which is used to extend the technique also for $\operatorname{dir}_{\max }$ pointing along the $x$ - or $y$-axis.

To exploit render-to-texture functionality along the z-axis, we render each slice from $S_{0}$ to $S_{\max }$ with texture coordinates reaching from $\left(0.0,0.0, z_{i}\right)$ to $\left(1.0,1.0, z_{i}\right)$, where $z_{i}$ is the $z$ coordinate of slice $S_{i}$ with $z_{i}=\frac{i}{\max }$. For each slice except $S_{0}$, we need to access the previously computed slice. Thus, during rendering each fragment corresponds to one voxel in the original data set and we can exploit a GLSL fragment shader to compute the lighting information as given by Equation 1. This is done based on the given cone angle described in Section 3. To compute both the scattering color as well as the shadowing intensity we apply back to front compositing to $S_{i}$ and $S_{i-1}$, as known from standard DVR. When assuming that the current voxel's color and opacity are stored as voxel.rgb resp. voxel.a, the scattering color $c\left(\vec{x}_{i}, \vec{\omega}\right)$ as well as the attenuated intensity $l_{i}\left(\vec{x}_{i}, \vec{\omega}\right)$ at $\vec{x}_{i}$ can be computed in the shader as follows:

$$
\begin{gathered}
c\left(\vec{x}_{i}, \vec{\omega}\right)=(1.0-\text { voxel.a }) * c\left(\overrightarrow{x_{i-1}}, \vec{\omega}\right)+\text { voxel.a } * \text { voxel.rgb }, \\
l_{i}\left(\vec{x}_{i}, \vec{\omega}\right)=(1.0-\text { voxel.a }) * l_{i}\left(\overrightarrow{x_{i-1}}, \vec{\omega}\right)+\text { voxel.a },
\end{gathered}
$$

whereby $c\left(\overrightarrow{x_{i-1}}, \vec{\omega}\right)$ and $l_{i}\left(\overrightarrow{x_{i-1}}, \vec{\omega}\right)$ are read around the intersection $\vec{p}_{i}$ of the current voxel's light vector $d \overrightarrow{i r_{l}}=\vec{x}_{l}-\vec{x}_{i}$ with the previous slice. Since in this step, we want to simulate the light traveling from the light source through the volume, the incident direction of $l_{i}\left(x_{\overrightarrow{i-1}}, \vec{\omega}\right)$ and the outgoing direction for $c\left(\vec{x}_{i}, \vec{\omega}\right)$ are the same. Thus, we can approximate the light traveling by considering the emission absorption model. While $l_{i}\left(x_{i-1}, \vec{\omega}\right)$ may be obtained by performing a single texture fetch at $\vec{p}_{i}$ if we want to guarantee hard shadows, $c\left(x_{i-1}, \vec{\omega}\right)$ is obtained by blending voxels in the neighborhood of $\vec{p}_{i}$. The amount of voxels and the blending factors are dependent on the cone angle $\theta$. We have implemented a Phong lobe phase function as shown in Section 3, where each contributing voxel is weighted with $\cos (\alpha)^{\beta}$, where $\alpha$ is the angle between the light vector from $\vec{x}$ and the vector towards the contributing voxel, and $\beta$ is a user specified fall-off coefficient. To achieve energy preservation, we further divide the computed contribution by the number of considered neighbors, using the previously introduced constant $C$.

In order to incorporate the scattering of the outgoing light, we can proceed similarly in a second pass. But instead of using $\vec{x}_{l}$ to determine $F_{0}$, we use the current camera position and set $d i \vec{r}_{\text {max }}$ to $\overrightarrow{n_{F_{0}}}$ before propagating illumination from back to front. During this propagation we blend the outgoing chromaticity with the previously computed incoming chromaticity and choose the minimum of the scalar luminance. While this proceeding is not physical correct, it leads to plausible results as discussed in Section 6.

Implementing the described light propagation scheme brings up some technical issues. First, as already mentioned, it is only possible to render into a 3D texture along the $\mathrm{z}$-axis. Therefore, the texture coordinates as assigned to the rendered slices are permutated based on a permutation vector, which stores for each axis in image space, to which axis it is mapped in volume space. Since this permutation is applied when generating the illumination volume, we have to apply its inverse to the texture coordinates when accessing this volume later on during rendering. Furthermore, for all texture fetches during the light propagation, it has to be ensured, that the information on the current slice $S_{i}$ is not taken into account. This can be either achieved by manually filtering in the $x y$-plane only, or more easily by exploiting texture arrays, which allow to filter only within one texture slice. Finally, when applying the described light propagation algorithm in cases, where $\vec{x}_{l}$ is located inside the volume, the lighting information cannot be propagated in a single direction. There are two alternative solutions for this 
problem. First, the lighting information can be propagated into both directions starting at $\vec{x}_{l}$. However, this approach may lead to noticeable discontinuities within the illumination volume. Therefore, we have decided to simply project $\vec{x}_{l}$ onto $F_{0}$ and apply the algorithm as described above in order to propagate lighting information into direction $\operatorname{dir}_{\text {max }}$. In our experiments this lead to results of sufficient quality.

While the illumination propagation can be done rapidly, since only basic operations are required, the four channel illumination volume might be a limitation when dealing with large data sets. When taking into account the perceptual properties of the human visual sense, we are able to reduce the memory footprint of the illumination volume. Therefore, we have exploited the fact, that chromaticity variations are less perceived than variations in luminance [13]. Keeping in mind, that the scattering is propagated through the $R G B$ channels and the attenuation through the $A$ channel, we are able to downscale the scattering volume by remaining the original size of the attenuation part. This is a technique, which has also been incorporated in the JPEG image compression technique, and it is referred to as chroma subsampling. In fact, instead of downsampling a previously generated data set, we directly modify the light propagation in order to also achieve a performance gain. However, since this increases the distance between the slices to process, the chromasubsampling factor $g_{c s}$ is used to modify the opacity of the current voxel prior to compositing: color. $a=$ color. $a * g_{c s}$. This avoids luminance variations when enabling chroma subsampling. The technique has been applied when rendering the salamander data set shown in Figure 4.

\subsection{Changing Light Position}

While the light propagation technique described in the previous subsection, would work well for arbitrary but fixed light positions $\vec{x}_{l}$, a dynamic change may introduce problems. This is due to the fact, that $d i \vec{r}_{\text {max }}$ runs always along one of the major volume axis. When $\vec{x}_{l}$ changes in a way that $\operatorname{dir}_{\max }$ is also changed, the illumination is propagated along a different direction through the volume, which might lead to noticeable popping artifacts.

To deal with this problem, we introduce an additional light propagation direction $d i \vec{r}_{\text {blend }}$. While $d i \vec{r}_{\text {max }}$ is the light propagation direction based on the cube face $F_{0}$ with the largest projection size as seen from $\overrightarrow{x_{l}}, d i \overrightarrow{r_{\text {blend }}}=-\overrightarrow{n_{F_{1}}}$ is based on $F_{1}$, the cube face with the second largest projection size (see Figure 3 ). When blending the results of the light propagation along these two principle axes, we can avoid popping effects, since the contribution of the possibly new $\operatorname{dir}_{\text {max }}$ has been already taken into account, before the the princi-

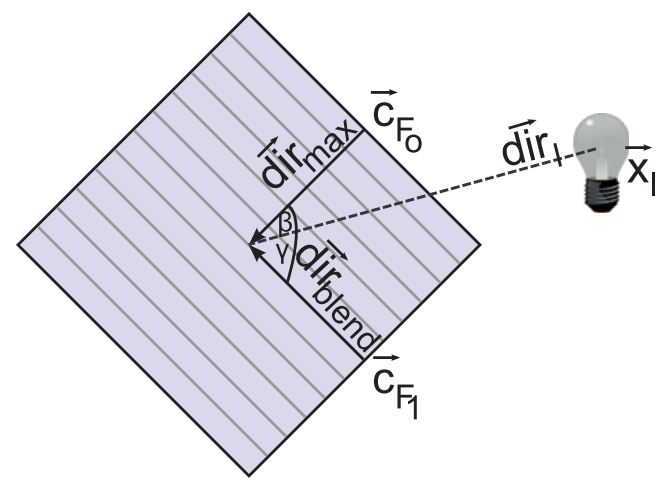

Figure 3: To avoid popping artifacts, when the main propagation axis $d i \vec{r}_{\text {max }}$ is changed, we employ a blending between the two principal light directions given by $F_{0}$ and $F_{1}$. ple light propagation direction has changed. Thus, the light volume generation is done, whereby the first propagation is along $\operatorname{dir}_{\text {max }}$ as described in Subsection 4.1, and the second is along $d i \overrightarrow{r_{b l e n d}}$. Therefore, we process the volume along $d_{i r_{b l e n d}}$ and compute the scattering and shadowing along this axis. But instead of using two different illumination volumes during rendering, we blend the computed illumination results $I_{1}$ and $I_{0}: S=\alpha_{0} \cdot I_{0}+\alpha_{1} \cdot I_{1}$.

As depicted in Figure 3, $\alpha_{0}$ and $\alpha_{1}$ are dependent on the angle $\beta$ between the light vector $d \overrightarrow{i r_{l}}$ and $d i \vec{r}_{\text {max }}$ resp. $\gamma$ between $d \overrightarrow{i r_{l}}$ and $\operatorname{dir}_{\overrightarrow{b l e n d}}$. To achieve an appropriate blending, we have to ensure that the sum of $\alpha_{0}$ and $\alpha_{1}$ always equals 1 . Furthermore, $\alpha_{1}$ must be 0 around the center area at $\overrightarrow{c_{0}}$, because otherwise popping would occur, when moving the light source over $\overrightarrow{c_{0}}$. Choosing $\alpha_{0}=1-$ $\frac{2 \cdot a \cos (\beta)}{\pi}$ and $\alpha_{1}=1-\frac{2 \cdot a \cos (\gamma)}{\pi}$ fulfills these requirements.

\section{Rendering}

Using current GPUs for rendering with our model is straight forward. While in principle the proposed lighting computation is applicable to any volume rendering technique, we use GPU-based volume ray-casting, whereby the shading is done with GLSL shaders. In the following, we assume the light volume has been generated as described in the previous section and is present as an $R G B A$ data set, where the $R G B$ channels represent the chromaticity $c(\vec{x}, \vec{\omega})$ and the $A$ channel the attenuated light intensity $l_{i}(\vec{x}, \vec{\omega})$, both at the current voxel's position $\vec{x}$. Such light volumes are shown in column (a) in Figure 4. Based on the values fetched from these data sets, we solve the front-to-back compositing volume rendering integral. Thus, shading of $\vec{x}$ is a multiplication of the transport color $t(\vec{x})$, as assigned through the transfer function, and $c(\vec{x}, \vec{\omega})$ to which we apply an intensity adaption based on $l_{i}(\vec{x}, \vec{\omega})$. In order to support emission, the emissive color $L_{e}(\vec{x})$ can be added. The compositing along the viewing ray is not altered.

Figure 4 shows the results, whereby (a) shows a volume rendering of the generated illumination volumes. From (b) to (d), the following techniques are shown. In (b) only shadowing is applied, whereby the voxel is assumed to be initially white. While we have used an $R G B A$ light volume for all cases shown in this section, a single channel shadow volume would have been sufficient for this shadowing only case. In (c), we only show the scattering color $c(\vec{x}, \vec{\omega})$ as computed during our volume processing. The figures labeled with (d) show the application of the entire illumination model combined with a specular term. Therefore, we multiply the transport color $t(\vec{x})$ and $c(\vec{x}, \vec{\omega})$, which is modulated based on $l_{i}(\vec{x}, \vec{\omega})$ as shown in Equation 1. While this rendering technique generates realistic results for diffuse and scattering materials, it is not possible to capture materials having a high degree of specularity. Therefore, we have combined the technique with an achromatic specular intensity $c_{S}(\vec{x})$ as calculated with the gradient-based Phong model. Since we assume, that the specular intensity is not wave length dependent we are able to multiply $c_{s}(\vec{x})$ and $l_{i}(\vec{x}, \vec{\omega})$, and are able to modulate the result by the gradient magnitude $|\nabla \tau(\vec{x})|$ in order to achieve the desired intensity:

$$
I=|\nabla \tau(\vec{x})| \cdot c_{s}(\vec{x}) \cdot l_{i}(\vec{x}, \vec{\omega}) .
$$

As it can be seen, surfaces appear to be vivid having a specular component, while diffuse elements are also present. Instead of modulating $c_{S}(\vec{x})$ with the gradient magnitude, it could also be modulated by the signal to noise ratio of the computed gradients. Thus, it would become possible to emphasize surfaces in an otherwise noisy data set.

It should be pointed out, that while we can use a single texture fetch in order to obtain $c(\vec{x}, \vec{\omega})$ as well as $l_{i}(\vec{x}, \vec{\omega})$ in the general case, we need to apply two texture fetches when exploiting the chroma subsampling introduced in Section 4. One in the original shadow 

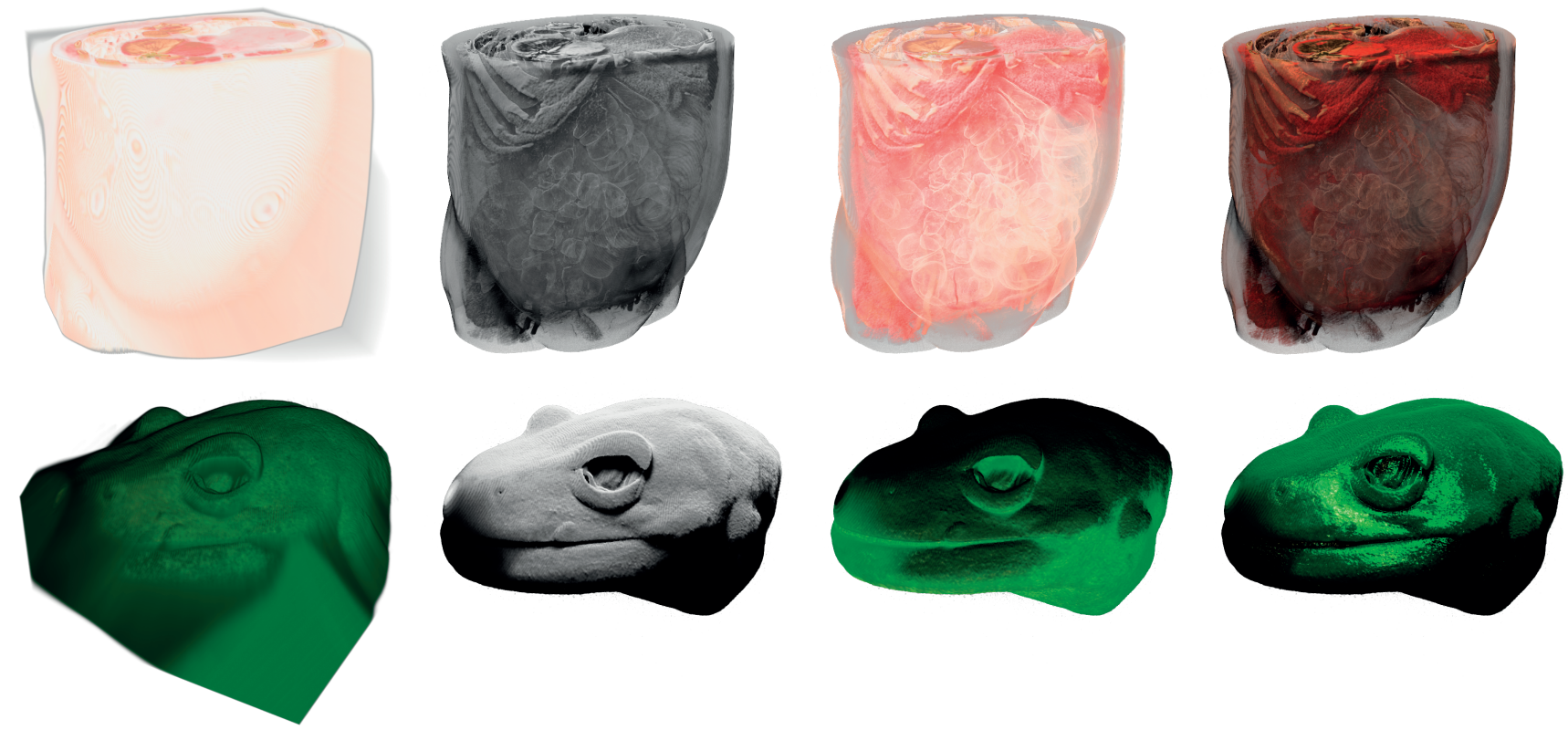

(a) illumination volume

(b) $l_{i}(\vec{x}, \vec{\omega})$

(c) $c(\vec{x}, \vec{\omega})$

(d) full shading

Figure 4: The generated illumination volume (a) can be used to achieve different rendering effects: rendering with the attenuated light intensity $l_{i}(\vec{x}, \vec{\omega})(\mathrm{b})$, with the scattering chromaticity $c(\vec{x}, \vec{\omega})(\mathrm{c})$ and with full shading as defined by our illumination model (d).
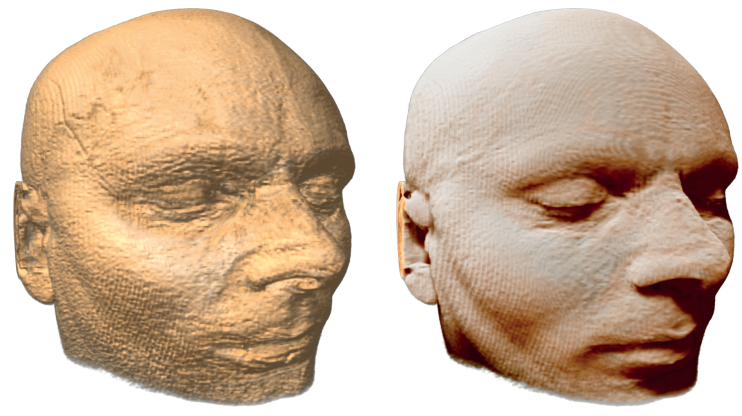

Figure 5: Comparison of an MRT scan of a human head $(256 \times 256 \times$ 256 voxels) rendered with gradient-based Phong illumination (left) and with our technique (right). The scattering contribution results in a more realistic appearance of the skin, while the shadowing supports the spatial comprehension.

volume and one being subject to a texture coordinate transformation into the scattering volume having a lower resolution.

\section{Results and Discussion}

Besides the application to CT data sets as shown in Figure 1 and Figure 4, we have also applied our algorithm to other modalities having a lower signal to noise ratio. Figure 5 shows the application to an MRT scan of a human head having a resolution of $256 \times 256 \times 256$ voxels. Compared to the gradient-based shading (left), our algorithm (right) results in a more realistic representation of the human skin, since scattering effects are taken into account. Furthermore, the shadowing gives the image more depth, which is especially visible below the nose and in the region around the eyes. We would like to point out the rather hard shadow borders, which would not be present when just applying scattering. While high quality shading of MRT data with conventional shading techniques is already challenging, it becomes nearly impossible when applying it to 3D US data, which suffers from an even lower signal to noise ratio (see Figure 6 (left)). When instead applying our technique to the same data set (see Figure 6 (right)), structures start to emerge and the spatial comprehension becomes more easy.

To analyze the performance of our algorithm, we have measured the fps over 1000 frames at a resolution of $1024 \times 1024$, where the rendered object is rotated around its up-vector. With this scenario, we have compared three different cases: Phong shading, and our technique with and without light source movement. For the Phong shading we have exploited forward difference gradients computed on the fly and have considered ambient, diffuse and specular illumination. Table 1 shows the results, we could achieve on a standard desktop computer, having an Intel Core2 Duo CPU E8400 running at $3.00 \mathrm{GHz}, 4 \mathrm{~GB}$ of main memory, and an nVidia GeForce GTX285 graphics board. As it can be seen, for most cases our technique results in higher frame rates than conventional shading. Only when recomputing the illumination volume, the frame rates drop below those achieved with gradient-based shading. The only case, where our technique was slower in both cases is the torso data set.

Since our volumetric illumination technique only approximates scattering and shadowing, we have decided to conduct a user study in order to assess its usefulness. To realize this, we have implemented an applet, with which each participant had to accomplish three different tests. Since the usefulness of a rendering algorithm is always dependent on the application case in which it is utilized and therefore hard to judge in general, we have decided to focus primarily on the facilitation of depth perception. This decision is based on the assumption, that depth perception is essential for most 3D viewing tasks. In each of the three tests, the participants have been confronted with numerous rendering results generated either with the technique described in this paper or alternatively with gradientbased Phong illumination. To achieve better comparability, each image has been incorporated twice into each test series, once rendered with our model and once with Phong. Besides from the rendering technique, all other parameters, e. g., transfer function as well as camera and light position, have been left unchanged. In the first test, the depth comparison test, 38 images have been shown to each participant. The goal of this test was to analyze relative 
depth perception. Therefore, two regions have been emphasized in each image by using circular markers and the user has been asked to click on the region, which is closer to the viewer. During this test we have measured the time used for each image as well as whether the correct region has been selected. In the second test, the depth estimation test, we wanted to judge absolute depth perception. To do so, we have emphasized only one region in each of the 14 shown images by using a spherical marker. In this test, the participants had to estimate the depth of the emphasized region in percentage of the overall depth extend of the shown object. The participants could input the estimated depth by using a slider as shown in Figure 7. In this test we have measured the difference between the real depth as computed via volume ray-casting and the estimated depth as transmitted using the slider. Additionally, we have also measured the time used for each image. As a third test, we have conducted a very subjective image comparison test, where we have shown 19 pairs of images to each participant. Again, both images where identical despite the used rendering technique. The subjects have been asked to select that image from the pair, which they find more visually pleasing. While the order of the three tests has been fixed for each participant (depth comparison, depth estimation, image comparison), the order of the displayed images has been randomized in order to reduce order-dependent side effects. In addition, each test started with a simple example in order to make the participants familiar with it. Since these examples where unambiguous, we have decided to omit the results for one test, if the participant failed doing the example correctly. This was never the case.

16 (age 22-33, $\varnothing: 27$ ) subjects participated in the study. Most subjects were students or members of the departments (computer science, mathematics, geoinformatics, and physics). All had normal or corrected to normal vision; 5 wear glasses or contact lenses. The time per subject including a short pre-questionnaire, reading the instructions, training, and experiment took approximately 8 minutes, where in total 94 images have been processed in the three different tests. The results of the depth comparison test are shown in Figure 8. In the top left bar chart, the average time used for conventional shading (3935.4 $\mathrm{ms}$ ) as compared to our technique $(3250.5 \mathrm{~ms})$ is shown. The lower left plot shows the percentage of correct depth decisions as based on conventional shading (71.4\%) and our technique $(94.2 \%)$. For both these results, we have performed a t-test and we found a significant $(\rho<0.01)$ increase in accuracy as well as speed, when using our technique for the relative depth perception in the tested cases. This is also reflected in the scatter plot shown in Figure 8 (right), where the average time needed and the percentage of correct depth decisions are plotted for each image. It can be clearly seen, that the images generated with our technique form a cluster in the top left region. Additionally, in

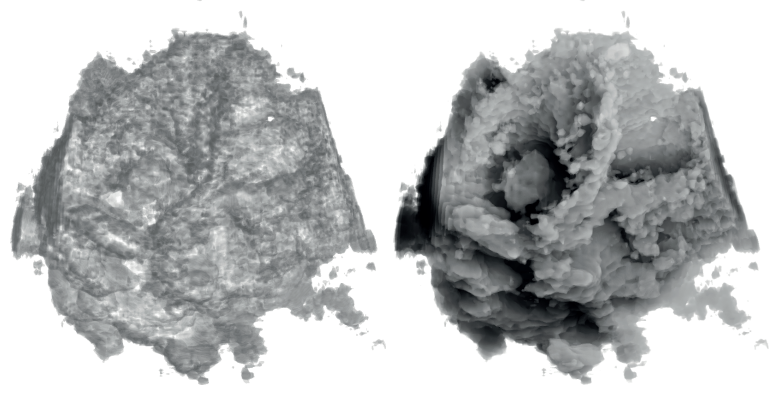

Figure 6: A 3D US scan of a human heart $(148 \times 203 \times 149$ voxels $)$ rendered with gradient-based Phong illumination (left) and with our technique (right). Due to the low signal to noise ratio, spatial comprehension is difficult when using gradient-based shading. Instead, when using our technique, structures emerge.

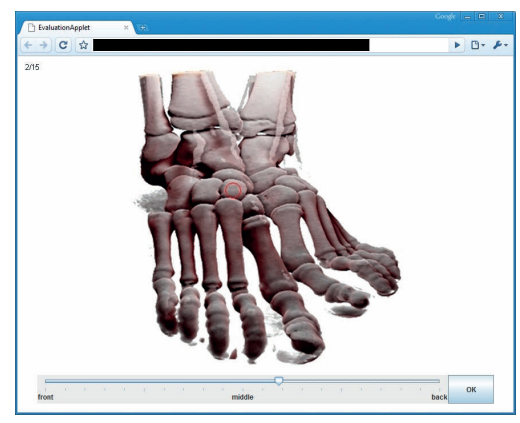

Figure 7: During the depth estimation test, the users had to estimate the depth of the highlighted region by adapting the slider, representing the entire depth extend of the shown object, accordingly.

the depth estimation test the average depth estimation was more accurate and it was less time used. However, based on the rather low number of subjects and images, we could not show a high significance. Finally, the results of the third test have shown, that our volumetric illumination model is perceived as more aesthetic in $72.4 \%$ of all shown cases.

\section{CONCLUSIONS AND FUtURE Work}

In this paper we have proposed a volumetric lighting model supporting scattering as well as shadowing effects, which allows interactive frame rates when implemented on current graphics hardware. We have derived the model and described our implementation based on illumination propagation. While the model is similar in spirit to the model proposed by Kniss et al. [11], it requires fewer sampling operations for the illumination calculation, since this is performed in volume space. This allows an easy integration into current state-of-the-art GPU-based volume ray-casters, which is not possible with the half-angle technique proposed by Kniss et al.. Based on our implementation, we have shown several successful application examples also including modalities with a low signal to noise ratio, as 3D US and MRT. Since the presented model and its implementation are physically-motivated but not physicallycorrect, we have conducted a user study, with which we have compared our technique to conventional gradient-based shading. Based on the result of three different tests, we were able to show, that the proposed model is able to improve the depth perception of volume renderings. Furthermore, the technique is very easy to implement, and can be easily combined with clipping planes and arbitrary classification techniques, while providing in many cases higher frame rates than gradient-based shading. This allows full control over all relevant rendering parameters as transfer function, lighting parameters and camera view. Therefore, we believe it has the potential to be integrated in other volume rendering frameworks.

Since we could show that the approximative nature of the model

Table 1: Performance of our technique with and without light volume regeneration as compared to local Phong illumination.

\begin{tabular}{|l|c|c|c|c|}
\hline data set & \multicolumn{2}{|c|}{$\begin{array}{c}\text { size } \\
\left(\text { voxel }^{3}\right)\end{array}$} & Phong & \multicolumn{2}{c|}{ our technique } \\
& $360 \times 270 \times 550$ & $12.6 \mathrm{fps}$ & $16.5 \mathrm{fps}$ & $7.2 \mathrm{fps}$ \\
mouse & $378 \times 346 \times 445$ & $11.3 \mathrm{fps}$ & $9.5 \mathrm{fps}$ & $4.2 \mathrm{fps}$ \\
torso & $512 \times 512 \times 202$ & $9.4 \mathrm{fps}$ & $17.3 \mathrm{fps}$ & $5.5 \mathrm{fps}$ \\
salamander & $256 \times 256 \times 256$ & $22.6 \mathrm{fps}$ & $25.0 \mathrm{fps}$ & $12.2 \mathrm{fps}$ \\
MRT head & 256 regen. & regen. \\
3D US heart & $148 \times 203 \times 149$ & $24.0 \mathrm{fps}$ & $31.4 \mathrm{fps}$ & $14.3 \mathrm{fps}$ \\
\hline
\end{tabular}



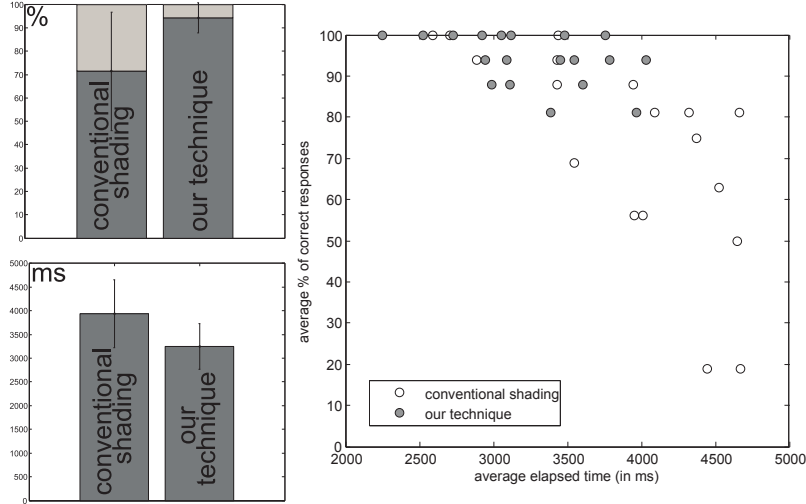

Figure 8: The results of the conducted depth comparison test. The plots on the left show the average time needed and the accuracy of depth decisions, while the scatter plot shows all images plotted based on their average time and accuracy. As it can be seen, images generated with our technique form a cluster towards more quick and accurate depth decisions.

still allows improved spatial comprehension, the major drawback is the usage of an additional illumination volume. However, by exploiting chroma subsampling, the situation can be improved. In addition to that, we are positive, that with the increasing memory size of graphics hardware, this will become even less important.

In the future, we would like to extend our technique in order to be able to integrate area light sources. Since our algorithm propagates illumination information from the outside of the volume inwards, it could be possible to project area light sources onto the border of the volume in order to achieve the desired effect. Additionally, it would be interesting to investigate the influence of multiple light sources. On the evaluation side, we would like to compare the shadowing to the ground truth and evaluate the influence of chroma subsampling.

\section{ACKNOWLEDGEMENTS}

This work was partly supported by grants from the Deutsche Forschungsgemeinschaft (DFG), SFB 656 MoBil Münster, Germany (project Z1). The presented concepts have been integrated into the Voreen volume rendering engine (www.voreen.org).

\section{References}

[1] K. M. Beason, J. Grant, D. C. Banks, B. Futch, and M. Y. Hussaini. Pre-computed illumination for isosurfaces. In Proceedings of the Conference on Visualization and Data Analysis (VDA), pages 1-11, 2006.

[2] U. Behrens and R. Ratering. Adding shadows to a texture-based volume renderer. In Proceedings of the IEEE International Symposium on Volume Visualization, pages 39-46, 1998.

[3] D. Dalby. Biological Illustration: A Guide to Drawing for Reproduction. Field Studies Council, 1980.

[4] P. Desgranges, K. Engel, and G. Paladini. Gradient-free shading: A new method for realistic interactive volume rendering. In Proceedings of the International Fall Workshop on Vision, Modeling, and Visualization (VMV), pages 209-216, 2005.

[5] Y. Dobashi, T. Yamamoto, and T. Nishita. Interactive rendering of atmospheric scattering effects using graphics hardware. In Proceedings of the ACM SIGGRAPH/EG Conference on Graphics Hardware (GH), pages 99-107, 2002.

[6] M. Hadwiger, A. Kratz, C. Sigg, and K. Bühler. GPU-accelerated deep shadow maps for direct volume rendering. In Proceedings of the ACM SIGGRAPH/EG Conference on Graphics Hardware (GH), pages 49-52, 2006.

[7] F. Hernell, P. Ljung, and A. Ynnerman. Efficient ambient and emissive tissue illumination using local occlusion in multiresolution vol- ume rendering. In Proceedings of the IEEE/EG International Symposium on Volume Graphics (VG), pages 1-8, 2007.

[8] F. Hernell, P. Ljung, and A. Ynnerman. Interactive global light propagation in direct volume rendering using local piecewise integration. In Proceedings of the IEEE/EG International Symposium on Volume and Point-Based Graphics (VG), pages 105-112, 2008.

[9] P. V. J. Daz, H. Yela. Vicinity occlusion maps - enhanced depth perception of volumetric models. In Proceedings of Computer Graphics International (CGI), pages 56-63, 2008.

[10] J. Kniss, S. Premoze, C. Hansen, and D. Ebert. Interactive translucent volume rendering and procedural modeling. In Proceedings of IEEE Visualization 2002, pages 109-116, 2002.

[11] J. Kniss, S. Premoze, C. Hansen, P. Shirley, and A. McPherson. A model for volume lighting and modeling. IEEE Transactions on Visualization and Computer Graphics, 9(2):150-162, 2003.

[12] M. S. Langer and H. H. Bülthoff. Depth discrimination from shading under diffuse lighting. Perception, 29(6):649-660, 2000.

[13] M. Livingston. Vision and Art: The Biology of Seeing. Harry N. Abrams, New York, 2002

[14] P. Ljung, F. Hernell, and A. Ynnerman. Local ambient occlusion in direct volume rendering. IEEE Transactions on Visualization and Computer Graphics, 15(2), 2009.

[15] T. Lokovic and E. Veach. Deep shadow maps. In SIGGRAPH 'O0: Proceedings of the 27th annual conference on Computer graphics and interactive techniques, pages 385-392, New York, NY, USA, 2000. ACM Press/Addison-Wesley Publishing Co.

[16] N. Max. Optical models for direct volume rendering. IEEE Transactions on Visualization and Computer Graphics, 1(2):99-108, 1995.

[17] A. Mendez, M. Sbert, and J. Cata. Real-time obscurances with color bleeding. In Proceedings of the Spring Conference on Computer Graphics (SCCG), pages 171-176, 2003.

[18] E. Penner and R. Mitchell. Isosurface ambient occlusion and soft shadows with filterable occlusion maps. In Proceedings of the IEEE/EG International Symposium on Volume and Point-Based Graphics (VG), pages 57-64, 2008.

[19] T. Ropinski, J. Kasten, and K. H. Hinrichs. Efficient shadows for GPU-based volume raycasting. In Proceedings of the International Conference in Central Europe on Computer Graphics, Visualization and Computer Vision (WSCG), pages 17-24, 2008.

[20] T. Ropinski, J. Meyer-Spradow, S. Diepenbrock, J. Mensmann, and K. H. Hinrichs. Interactive volume rendering with dynamic ambient occlusion and color bleeding. Computer Graphics Forum (Eurographics 2008), 27(2):567-576, 2008.

[21] M. Ruiz, I. Boada, I. Viola, S. Bruckner, M. Feixas, and M. Sbert. Obscurance-based volume rendering framework. In Proceedings of the IEEE/EG International Symposium on Volume and Point-Based Graphics $(V G)$, pages 113-120, 2008.

[22] C. R. Salama. GPU-based monte-carlo volume raycasting. In Proceedings of Pacific Graphics (PG), 2007.

[23] M. Schott, V. Pegoraro, C. Hansen, K. Boulanger, and K. Bouatouch. A directional occlusion shading model for interactive direct volume rendering. In Computer Graphics Forum (Proceedings of Eurographics/IEEE VGTC Symposium on Visualization 2009), pages 855-862, 2009.

[24] A. J. Stewart. Vicinity shading for enhanced perception of volumetric data. In Proceedings of IEEE Visualization (VIS), page 47, 2003.

[25] J. Sun and P. Perona. Where is the sun? Nature Neuroscience, (1):183-184, 1998.

[26] L. Wanger. The effect of shadow quality on the perception of spatial relationships in computer generated imagery. In SI3D '92: Proceedings of the 1992 symposium on Interactive 3D graphics, pages 39-42, 1992.

[27] C. Zhang and R. Crawfis. Volumetric shadows using splatting. In Proceedings of IEEE Visualization (Vis), pages 85-92, 2002.

[28] C. Zhang and R. Crawfis. Shadows and soft shadows with participating media using splatting. IEEE Transactions on Visualization and Computer Graphics, 9(2):139-149, 2003.

[29] S. Zhukov, A. Iones, and G. Kronin. An ambient light illumination model. In Proceedings of the EG Workshop on Rendering (EGRW), pages $45-55,1998$. 